\title{
Collaborative Concept Mapping as a Pre-Writing Strategy for L2 Learning: A Korean Application
}

\author{
Yongjin Lee
}

\begin{abstract}
This study aimed at examining the use of concept mapping technique in a course module for Korean language learning with U.S. college students, who were prompted to engage in collaborative writing planning. The study assumed that concept mapping enhances students' communicative interaction to promote their L2 writing. One hundred and twenty-three participants were recruited from three different proficiency level classes (beginning, intermediate, and advanced classes). Data were collected during three writing sessions: pretest of writing, individual planning, and collaborative planning. The MANCOVA results on the five components of composition score showed that the effect of collaborative concept mapping on $L 2$ writing significantly differed across the three class levels. The study suggested practical implications for the use of collaborative mapping tasks in L2 writing contexts.
\end{abstract}

Index Terms-Collaborative language learning, concept mapping, prewriting strategy, second language writing learning.

\section{INTRODUCTION}

Concept mapping is a strategy to represent a student's own meaning making process during learning [1]. A concept map consists of nodes and labeled connective lines to externalize concepts and propositions learned. In the 1970s, Novak and his research groups started to study the concept mapping technique to measure the structure and organization of a learner's knowledge [2]. The concept mapping has been used in various educational settings, and generally has positive effects on both knowledge attainment and attitude [3]. In first language learning contexts, it has been reported that concept mapping is beneficial in facilitating the writing process [4], [5]. However, empirical research on concept mapping as a prewriting strategy in second language (L2) learning contexts is limited.

\section{A. Collaborative Concept Mapping}

Concept mapping is a reflective process and emphasizes the reasons and rationales of associations with related concepts. In the initial stage of concept mapping process, students are expected to focus more on their memorized vocabulary and cultural knowledge related to the given topic, rather than on complete sentence structures. Further the concept mapping activity has students feel less concerned about making mistakes, and provoke students to develop ideas, words, concepts or statements on a writing topic.

Manuscript received September 25, 2012; revised January 15, 2013.

Yongjin Lee was with the Instructional Design, Development and Evaluation Department, Syracuse University, Syracuse, NY 13244 USA (e-mail: eduist@ gmail.com).
Students can also elaborate various perspectives by attempting to develop more comprehensive concept maps [6]. The structural format of concept maps may help students' cognitive processing channels which enhance main idea concepts and aid the organization of ideas for recall [7].

Research has suggested the potential of collaborative concept mapping [8], [9]. The underlying assumption was that collaborative concept mapping would be supportive of generating discussions beneficial to learning. Through constructing a group concept map, group members explain their views and knowledge to one another about a topic. The group members also negotiate and develop collaboratively the meanings they would add to their concept maps [10], [11].

Okebukola's study [9] demonstrated that the students who were engaged in cooperative learning assisted by concept mapping activities outperformed those who worked on their concept maps individually. De Simone, Schmid, and McEwen [12] asserted that the mapping process allowed learners to manage, construct, and share their own understanding of the content from an ill-structured domain. Gilbert and Greene [13] also expected that collaborative concept mapping can facilitate higher-order thinking. But their study showed that the changes reflected in the maps were not necessarily the result of the group collaboration.

Based on previous studies presenting positive effect of collaborative concept maps in education settings (i.e., [8], [14]), this study hypothesized that collaborative concept mapping technique enhance communicative interactions among language learners, and promote their composition process.

\section{B. Collaborative Prewriting Strategy for L2 Learning}

Many previous studies in language instruction have focused on collaborative learning experiences [15]-[17]. The collaborative language learning strategies focus on enhancing communicative competences rather than rote memorization of grammar rules. In particular, collaborative strategies in planning stage of writing may facilitate meaningful interaction among the diverse learners, possibly resulting in increased language proficiencies for $\mathrm{L} 2$ learning

Writers use prewriting strategies in collecting information and reflecting upon it in the very early stage of writing. Planning strategies and activities reduce constrains which may include unorganized memories and propositions, linguist conventions of written tests, and rhetoric problems [18]. That is, the planning with appropriate prewriting strategies enhances the writer's retrieval and application of knowledge. In the planning stage of writing, the use of map format such as flow charts, trees, boxes, arrows and other 
notes ease the cognitive load on attention and working memory, yet allow the writer to keep moving [18]. Schulz [19] argued that the mapping process helps language learners to visualize their ideas as multidimensional constructs that imitate the movement of thought itself.

It is also important to note that collaborative prewriting strategy might benefit for heterogeneous groups of language learners. The foreign language learners in higher education settings in the United States are very diverse in terms of their cultural experiences, language proficiencies, attitudes and motivation toward target language and culture. Such individual differences are important factors that can affect language learning outcomes [20]. Webb [21] suggested that mixed-ability groups of students produce more interactions for collaborative tasks and higher achievement than uniform-ability groups. The collaborative prewriting task may prompt to use new vocabularies to generate concepts, to negotiate meanings of the concepts and their relationships based on the shared ideas among group members. For such learning activities, tasks should be presented in genuine situations that allow language learners to use their pre-constructed knowledge. Eventually they can become more aware of the target language culture and connotations for real life purposes.

However, the impact of prewriting strategies on students' writing improvement has been reported as mixed [18], [22]. The evidence of the effect of collaborative planning on L2 writing is not clear, although a collaborative language learning process has been emphasized in language programs [23]. This study explored the effect of collaborative concept mapping tasks as a pre-writing strategy in a L2 learning context. Further, the practical implications are suggested to help language teachers and instructional designers adopt the concept mapping strategies in L2 programs.

Thus, the specific research questions included: (1) is there a significant difference in composition profile scores between students who use concept maps for their writing planning and those who do not use concept maps for planning?; (2) is there a significant difference in composition profile scores between individual writing session and collaborative writing session?; and (3) is there a significant interaction of concept mapping treatment, writing session, and class level on the composition profile scores?

\section{METHOD}

To accomplish the research goal, this study employed a quasi-experimental repeated measure design, which is one of the most powerful designs [24]. As the within-subject variable, three writing sessions were investigated: the treatment group had a pre-test of writing, the first writing session using individual concept maps, and the second writing session using collaboratively-constructed concept maps, while the comparison group had a pre-test of writing, and two writing sessions without using concept mapping strategy.

\section{A. Participants}

One hundred thirty two English-speaking university students registered in Korean courses at a university in the
Northeastern United States were recruited for treatment groups and comparison groups. The sample school is a mid-sized, four-year higher educational institution which offers three different proficiency-level Korean courses including beginning, intermediate, and advanced classes. The treatment group and the comparison group included three different level classes. The complete data produced from one hundred twenty three participants (treatment $n=63$, comparison $n=60$ ) after list-wise deletion of the missing data were used in analysis.

\section{B. Procedures}

Since this study employed a nonequivalent comparison group design in which the treatment and comparison group were not randomly assigned, the pre-assessment of pre-learned writing skill was used for statistical adjustment of the nonequivalence between groups. The pretest was a 50-min in-class writing session for both group students to generate a narrative essay based on the given writing prompt.

The writing prompts used in this study were related to everyday topics such as school experiences, foods, fun activities at a party, and so on. Two experts in Korean language and literature field in the university reviewed writing prompts and modified the tasks. Within a level of Korean proficiency, the treatment and comparison groups were provided with same writing prompt and writing instruction.

One week after the pre-assessment, the treatment groups participated in the 50-minute concept mapping training sessions. The training involved a presentation of concept mapping techniques, followed by guided practice sessions. The researcher introduced the general idea of concept mapping techniques and the methods for constructing concept maps and using maps as a pre-writing strategy. Both treatment and comparison groups were also given the 15-minute writing instruction. The researcher provided all participants with handouts of the composition rubrics and briefly explained the categories of composition scores based on the rubrics. The researcher emphasized the planning process of writing by explaining that the writing process involves multiple tasks such as planning, drafting, and editing.

In the following two weeks, the treatment groups were encouraged to use concept maps to plan their compositions in the two writing sessions (individual planning vs. collaborative planning). At the first writing session, both group students were encouraged to spend some time to plan individually their compositions and develop their narrative essay. One week after the individual writing session, the treatment group students were randomly assigned to three-person groups to construct their group concept maps to plan their composition. Based on the group map, individuals developed their essay. At the second writing session, the comparison group students were also assigned to three-person groups to discuss the writing topic and individually develop their essay based on their discussion. Both groups had 70 minutes to finish planning and writing their essay at each writing session.

\section{Composition Scoring Rubrics}

To measure the quality of students composition, a rubric 
was adapted from Jacobs, Zinkgraf, Wormuth, Hartfiel, and Hughey [25]'s Composition Profile. The original Composition Profile [25] tends to assess various aspects of L2 learners' writing ability and is widely used as reliable analytic scales in the ESL field [26]. The measures cover five components of writing performance including content, organization, vocabulary, language use, and mechanics, and weighted according to its approximate importance for written communication [25]. Two experienced instructors scored the collected essays during the one-week period following the data collection. Before scoring the data, the raters discussed the adapted rubrics and resolved their questions. Based on the consensus of criteria, the raters' scoring was pilot-tested to determine inter-rater reliability coefficients (Cronbach's $\alpha$ $=.966)$.

\section{RESULTS}

To analyze the treatment effects on the specific aspects of composition, a three-way, repeated measure multivariate analysis of covariance (MANCOVA) with treatment condition, class, and writing session as the variables was performed on the five component scales (Content, Organization, Language Use, Vocabulary, and Mechanics), with the corresponding pre-test scores serving as covariates.

With regard to the first research question, the multivariate tests indicated that there were significant main effects for group (treatment), $F(5,107)=12.888$. This main effect indicated that the treatment group students who have used concept maps for their writing planning scored significantly higher than did the comparison group students on the five composition scales. The follow-up, univariate analyses of covariance revealed significant differences between treatment and comparison groups for Content, $F(1,111)=$ $31.449, p<.001$, Organization, $F(1,111)=28.607, p<.001$, and Vocabulary scores, $F(1,111)=15.071, p<.01$. The significant findings indicated that the concept mapping treatment group students scored significantly higher than did comparison group students on Content, Organization, and Vocabulary measures at both writing sessions.

Univariate $F$ tests also presented that there were significant two-way interactions of group and class for Organization, $F(2,111)=4.584, p<.025$, and Vocabulary measures, $F(2$, $111)=5.799, p<.01$. The result indicated that the concept mapping treatment effects on the measures of Organization and Vocabulary were significantly different among classes at both writing sessions.

The second research question concerns the effect of collaboration in writing planning on the composition scores. Multivariate tests revealed no significant main effect of session (individual vs collaborative planning) for the composition subscales, $F(5,107)=1.129, p=.350$. That is, students' composition scores on the five subscales were not significantly changed across writing sessions.

For the third question regarding to the interaction effects, there were significant interactions of session and class, $F(10$, $216)=2.214, p<.025$, and of session, group, and class, $F(10$, $216)=6.739, p<.001$. The univariate $F$ tests indicated that there was a significant two-way interaction of session and class for Vocabulary, $F(2,111)=4.701, p<.025$, and significant three-way interactions of session, group, and class for Content, $F(2,111)=7.034, p<.01$, and Language Use, $F(2,111)=5.816, p<.01$, as shown in Table I.

TABLE I: UNIVARIATE AND MUlTIVARIATE TESTS OF COLLABORATIVE SESSION EFFECT

\begin{tabular}{|c|c|c|c|c|c|c|c|}
\hline \multirow[t]{2}{*}{ Source } & \multicolumn{4}{|l|}{ Univariate } & \multicolumn{3}{|c|}{ Multivariate } \\
\hline & Measure & $\mathrm{F}$ & Sig. & Par. Eta ${ }^{2}$ & F & Sig. & Par. Eta ${ }^{2}$ \\
\hline \multirow[t]{5}{*}{ Session } & Content & 1.315 & .254 & .012 & 1.129 & .350 & .050 \\
\hline & Organization & .472 & .493 & .004 & & & \\
\hline & Vocabulary & .455 & .502 & .004 & & & \\
\hline & Lang. Use & .083 & .774 & .001 & & & \\
\hline & Mechanics & 4.752 & .031 & .041 & & & \\
\hline \multirow{5}{*}{$\begin{array}{l}\text { Session } \\
\mathrm{x} \\
\text { Group }\end{array}$} & Content & .817 & .368 & .007 & .578 & .716 & .026 \\
\hline & Organization & .130 & .719 & .001 & & & \\
\hline & Vocabulary & .070 & .792 & .001 & & & \\
\hline & Lang. Use & .698 & .405 & .006 & & & \\
\hline & Mechanics & 1.008 & .317 & .009 & & & \\
\hline \multirow{5}{*}{$\begin{array}{l}\text { Session } \\
\mathrm{x} \text { Class }\end{array}$} & Content & 3.440 & .036 & .058 & 2.214 & $.018 *$ & .093 \\
\hline & Organization & 3.111 & .048 & .053 & & & \\
\hline & Vocabulary & 4.701 & $.011 *$ & .078 & & & \\
\hline & Lang. Use & 2.243 & .111 & .039 & & & \\
\hline & Mechanics & .998 & .372 & .018 & & & \\
\hline \multirow{5}{*}{$\begin{array}{l}\text { Session } \\
\mathrm{x} \\
\text { Group } \\
\mathrm{x} \\
\text { Class }\end{array}$} & Content & 7.034 & $.001 * *$ & .112 & 6.739 & $.000 * *$ & .238 \\
\hline & Organization & .168 & .845 & .003 & & & \\
\hline & Vocabulary & 2.072 & .131 & .036 & & & \\
\hline & Lang. Use & 5.816 & $.004 * *$ & .095 & & & \\
\hline & Mechanics & 1.076 & .344 & .019 & & & \\
\hline
\end{tabular}

Because of inflated Type I error rate due to the multiple comparisons [27], a Bonferroni type adjustment for pairwise comparisons was used to explore the differences between all variables. The pairwise comparisons demonstrated that the treatment group outperformed the comparison group on Content, Organization, and Vocabulary. When students used group concept maps for planning, their composition scores for Content, Vocabulary, Language Use, and Mechanics were slightly increased. Specifically, Language Use and Mechanics scores from collaborative writing session were higher than those from individual writing session, and the mean differences of the two sessions were statistically significant. In spite of this mean difference, univariate $F$ test data suggested that overall students' compositions were not much improved in collaborative writing session.

\section{DISCUSSION}

The study findings are consistent with previous studies that reported a positive impact of concept mapping on the L2 learning (i.e., [19]-[30]). The concept mapping strategy can be used as a prewriting strategy that serves as a memory aid to lessen the attentional overload during composing activities [18]. Concept mapping activities can also help beginning writers unblock their thinking process to complete the writing task, and allow them better monitor higher level writing processes [31].

Specifically, the findings showed that the Content, Organization, and Vocabulary scores of the students who 
used concept mapping strategy for their writing were significantly higher than those of the comparison group students. The Content component primarily focuses on generating valid ideas. This study elicited that the concept mapping treatment group performed better on generating ideas than did the comparison group. Lin's study [29] also reported that a concept mapping activity was beneficial for generating ideas in persuasive writing. The concept mapping strategy in this study may have helped the language learners focus their attention on the topic to choose appropriate ideas for their assigned writing tasks.

The concept maps could have been supportive 'semantic organizers' [32] helping students become adept at organizing their ideas for their composition and assisting them in developing more comfort in their own writing abilities. These results support the notion that a concept mapping process may help students focus and organize relevant information primarily at a semantic level before starting an orderly writing activity [32].

In addition, with regard to Language Use and Mechanics scale, the treatment group's scores were also slightly higher than the comparison group's scores, although the mean differences were not statistically significant. Thus, concept mapping activities may have the potential to have significant impact on quality of writing when used as a learning activity in a prewriting phase of compositions.

In this study, the collaborative concept mapping activities at the prewriting stage could afford students the opportunity to pool ideas on the task. However, because of the limited time for collaborative planning, the peer interactions may not have been sufficient to allow students to have joint responsibility for completing the concept mapping task. Students seem to spend more time on individually writing their text. For successful collaboration in writing classes, the challenge is how to promote peer interactions that build group responsibility to complete writing tasks [33].

To promote peer interactions, collaborative concept mapping activities could also be designed differently. As Novak [1] suggested, students can be encouraged to individually develop a concept map first, then share their concept maps with group members to construct a group concept map. After constructing individual concept maps, members of a group may then work together to seek better ways to organize and represent knowledge on the given topic. These types of learning activities may allow students to spend more time thinking either in or about the target language. They may also encourage students to critically review others concept maps and engage peers in negotiating meanings of concepts and propositions. The shared concept maps may afford students the opportunities to see a view of the larger conceptual picture of the topic being written about [1], thus enhancing their L2 skills and confidence in their new knowledge. Further investigation is required to explore whether the kinds of collaborative concept mapping activities that may be appropriate for the planning process in L2 writing.

It was also noticeable that the effects of collaborative concept mapping treatment on the final composition were different according to the class levels. The statistical data illustrated that the treatment group of beginning classes achieved higher scores from the collaborative writing session than those from the individual writing session. Meanwhile, the intermediate and advanced class had mixed results in terms of improvement by collaborative planning for the five subscales of composition. The upper level students did not seem to benefit from collaboration for their final composition as much as the beginning class.

This finding raised a question as to which variables in grouping of classes influenced the effect of collaborative activities for writing planning. The class variable represents heterogeneity of subjects in terms of cultural backgrounds as well as language proficiency. The beginning class included heritage students who have a family background in which Korean language is, or was, spoken and non-heritage students who are learning Korean as a foreign language. Even in the heritage learners group, they were varied in their strengths in Korean language and their cultural backgrounds. Students in intermediate and advanced classes were all heritage language learners.

Literature has suggested that the heritage language learner group is more advanced than the non-heritage learner group in target language skills [34]. In the beginning classes, more advanced heritage learners are likely to have been able to help the foreign language learners with lower proficiency. Since heritage language learners seem to have been using more extensive contacts with the target language and culture, especially with family members and for entertainment [34], they were probably able to carry out the writing tasks more easily than the foreign language learners. However, some studies reported a negative impact on high-ability students when paired in heterogeneous dyads [21]. The influence of heterogeneous grouping with heritage language learners and foreign language learners in collaborative activities on language learning needs to be investigated further.

\section{CONCLUSION}

The study findings provided the evidence that concept mapping technique is a viable strategy to help L2 learners' writing planning process, thus ultimately improving their composition. However, the peer collaboration for constructing concept maps did not support improvements in composition scores. Considering the findings of this study as well as those of previous L2 writing research (i.e., [19], [30]), language teachers and instructional designers should focus on providing strategic devices such as concept mapping applicable to specific phases of writing process and monitoring how L2 learners use them in their practice.

Specifically, the peer's communicative interactions in concept mapping process should be observed. Despite literature that has suggested the notion that peer collaboration provides meaningful learning opportunities in which students can build their own knowledge structure in language learning contexts (i.e., [16], [17], [23]), this study did not support that conclusion. In particular, group dynamics and interaction patterns in manipulating group concept maps might be an important factor to influence the success of collaborative tasks for writing. Group members' conflicts in completing collaborative tasks may obstruct the students' learning [35]. 
When students devoted a large amount of time negotiating social protocols to concurrently manipulate the nodes and link objects of a shared concept map, they could have little time to concentrate directly on knowledge-related discussions [35]. When the students fully understand the collaborative writing process and have joint responsibility to the collaborative writing product, they can take much more benefit from the group concept mapping activity to improve their writing. Writing teachers may need to provide instructional guides for promoting peer interactions and monitor the collaborative working process. Developing logistics to promote peer interactions in meaningful ways to construct group maps will be an important direction in further investigation of collaborative concept mapping process in writing classes.

\section{REFERENCES}

[1] J. D. Novak, Learning, creating, and using knowledge: Concept Maps $^{T M}$ as facilitative tools in schools and corporations, Mahwah, NJ: Lawrence Erlbaum Associates, 1998.

[2] E. Plotnick, "Concept mapping: A graphical system for understanding the relationship between concepts," ERIC Digest, 1997.

[3] J. C. Nesbit and O. O. Adesope, "Learning with concept and knowledge maps: A meta-analysis," Review of Educational Research, vol. 76, no. 3, pp. 413-430, 2006.

[4] F. Pieronek, "Using maps to teach note taking and outlining for report writing," The Social Studies, vol. 85, pp. 165-169, 1994.

[5] S. B. Reynolds and J. Hart, "Cognitive mapping and word processing: aids to story revision," Journal of Experimental Education, vol. 58, pp. 273-279, 1990.

[6] N. Stoyanova and P. Kommers, "Concept mapping as a medium shared cognition in computer-supported collaborative problem solving," Journal of Interactive Learning Research, vol. 13, no. 1-2, pp. 111-133, 2002.

[7] A. M. O'Donnell, F. D. Dansereau, and R. H. Hall, "Knowledge maps as scaffolds for cognitive processing," Educational Psychology Review, vol. 14, no. 1, pp. 71-86, 2002.

[8] G. Hughes and D. Hay, "Use of concept mapping to integrate the different perspectives of designers and other stakeholders in the development of e-learning materials," British Journal of Educational Technology, vol. 32, no. 5, pp. 557-569, 2001.

[9] P. A. Okebukola, "Attitude of teachers toward concept mapping and vee diagramming as meta-learning tools in science and mathematics," Educational Research, vol. 34, no. 3, pp. 201-213, 1992.

[10] W. M. Roth and A. Roychoudhury, "The social construction of scientific concepts or the concept map as conscription device and tool for social thinking in high school science," Science Education, vol. 76, pp. 531-557, 1992.

[11] S. Sizmur and J. Osborne, "Learning processes and collaborative concept mapping," International Journal of Science Education, vol. 19 no. 10, pp. 1117-1135, 1997.

[12] C. De Simone, R. F. Schmid, and L. A. McEwen, "Supporting the learning process with collaborative concept mapping using computer-based communication tools and processes," Educational Research and Evaluation, vol. 7, no. 2-3, pp. 263-283, 2001.

[13] N. J. Gilbert and B. A. Greene, "College student's collaborative use of inspiration to generate concept maps in an educational technology class," Journal of Educational Technology Systems, vol. 30, no. 4, pp. 389-402, 2002.

[14] L. A. Freeman and L. M. Jessup, "The power and benefits of concept mapping: measuring use, usefulness, ease of use, and satisfaction," International Journal of Science Education, vol. 26, no. 2, pp. 151-169, 2004.

[15] M. Calderon, R. Hertz-Lazarowitz, and R. E. Slavin, "Effects of bilingual cooperative integrated reading and composition on students making the transition from Spanish to English reading," Elementary School Journal, vol. 99, pp. 153-165, 1998.
[16] Z. Dörnyei, "Psychological processes in cooperative language learning: Group dynamics and motivation," The Modern Language Journal, vol. 81, no. 4, pp. 482-493, 1997.

[17] T. Lehtonen and S. Tuomainen, "CSCL- a tool to motivate foreign language learners: The Finnish application," ReCALL, vol. 15, no. 1, pp. 51-67, 2003.

[18] R. T. Kellogg, "Effectiveness of prewriting strategies as a function of task demands," American Journal of Psychology, vol. 103, no. 3, pp. 327-342, 1990

[19] J. M. Schultz, "Mapping and cognitive development in the teaching of foreign language writing," The French Review, vol. 64, no. 6, pp. 978-988, 1991.

[20] R. Rueda and C. B. Chen, "Assessing motivational factors in foreign language learning: Cultural variation in key constructs," Educational Assessment, vol. 10, no. 3, pp. 209-229, 2005.

[21] N. M. Webb, "Student interaction and learning in small groups," Review of Educational Research, vol. 52, pp. 421-445, 1982.

[22] R. B. Kozma, "The impact of computer-based tools and embedded prompts on writing processes and products of novice and advance college writers," Cognition and Instruction, vol. 8, no. 1, pp. 1-27, 1991.

[23] R. L. Oxford, "Cooperative learning, collaborative learning and interaction: Three communicative strands in the language classroom," The Modern Language Journal, vol. 81, no. 4, pp. 443-456, 1997.

[24] K. Weinfurt, "Multivariate analysis of variance," in L. Grimm and P. Yarnold, Eds., Reading and understanding multivariate statistics, Washington. D.C: APA, 1995.

[25] H. L. Jacobs, S. A. Zinkgraf, D. R. Wormuth, V. F. Hartfiel, and J. B. Hughey, Testing ESL composition: A practical approach, Rowley, MA: Newbury House, 1981

[26] S. C. Weigle, Assessing Writing, Cambridge: Cambridge University Press, 2002.

[27] B. G. Tabachinick and L. S. Fidell, Using multivariate statistics, 4th eds. Needham Heights, MA: Allyn and Bacon, 1996.

[28] G. S. Bahr and D. F. Dansereau, "Bilingual knowledge maps (BiK maps) as a presentation format: delayed recall and training effects," The Journal of Experimental Education, vol. 73, no. 2, pp. 101-118, 2005.

[29] S.-Y. Lin, "The effects of computer-based concept mapping as a prewriting strategy on the persuasive writing of eighth-graders at a middle school in southeastern Idaho," Ph.D. dissertation, Idaho State University, 2003.

[30] M. Ojima, "Concept mapping as pre-task planning: A case study of three Japanese ESL writers," System, vol. 34, pp. 566-585, 2006.

[31] J. M. Sturm and J. L. Rankin-Erickson, "Effects of hand-drawn and computer-generated concept mapping on the expository writing of middle school students with learning disabilities," Learning Disabilities Research and Practice, vol. 17, no. 2, pp. 124-139, 2002.

[32] R. S. Pehrsson, and H. A. Robinson, The semantic organizer approach to writing and reading instruction, Rockvill, ML: Aspen Publishers, Inc., 1985

[33] N. Storch, "Collaborative writing: Products, process, and students' reflections," Journal of Second Language Writing, vol. 14, pp. 153-173, 2005.

[34] K. Kondo-Brown, "Differences in language skills: heritage language learner subgroups and foreign language learners," The Modern Language Journal, vol. 89, no. 4, pp. 563-581, 2005.

[35] C.-H. Chiu, "Evaluating system-based strategies for managing conflict in collaborative concept mapping," Journal of Computer Assisted Learning, vol. 20, pp. 124-132, 2004.

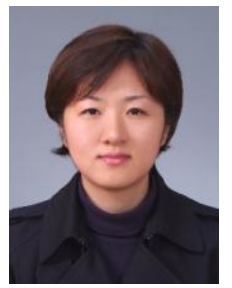

Yongjin Lee is a postdoctoral research fellow in the Department of Educational Technology of Hanyang Unversity in Seoul, South Korea. She received a M.A. in educational psychology from Korea University in Seoul, South Korea in 1998, and a Ph.D. in the department of instructional design, development, and evaluation from Syracuse University in New York, USA, 2010. She has some teaching experiences in higher education settings (e.g., the State University of New York at Binghamton, Korea University, etc.). Also she served as a director at Koreanische Schule in Bochum, Germany in 2011. In 2012, she joined Hanyang University. 